\title{
Flux across microneedle-treated skin is increased by increasing charge of naltrexone and naltrexol in vitro
}

\author{
Stan L. Banks ${ }^{a}$, Raghotham R. Pinninti ${ }^{a}$, Harvinder S. Gill ${ }^{b}$, Peter A. Crooks ${ }^{a}$, Mark R. \\ Prausnitz $^{\mathrm{b}, \mathrm{c}}$, and Audra L. Stinchcomb ${ }^{\mathrm{a}}$ \\ aDepartment of Pharmaceutical Sciences, University of Kentucky College of Pharmacy, Lexington, \\ Kentucky 40536-0082 \\ bThe Wallance Coulter School of Biomedical Engineering at Georgia Tech and Emory University, \\ Georgia Institute of Technology, Atlanta, GA 30332-0363 \\ 'School of Chemical and Biomolecular Engineering, Georgia Institute of Technology, Atlanta, GA \\ 30332-0100.
}

\begin{abstract}
Purpose-The purpose of this investigation was to evaluate the in vitro microneedle (MN) enhanced percutaneous absorption of naltrexone hydrochloride salt $(\mathrm{NTX} \cdot \mathrm{HCl})$ compared to naltrexone base (NTX) in hairless guinea pig skin (GP) and human abdominal skin. In a second set of experiments, permeability of the major active metabolite 6- $\beta$-naltrexol base (NTXOL) in the primarily unionized (unprotonated) form at $\mathrm{pH} 8.5$ was compared to the ionized form ( $\mathrm{pH} 4.5)$.

Methods-In vitro fluxes of NTX, NTX·HCl and ionized and unionized NTXOL were measured through microneedle treated or intact full thickness human and GP skin using a flow through diffusion apparatus. Solubility and diffusion samples were analyzed by HPLC.

Results-Both GP and human skin show significant increases in flux when treated with $100 \mathrm{MN}$ insertions as compared to intact full thickness skin when treated with NTX $\mathrm{HCl}$ or ionized NTXOL (pH 4.5) ( $\mathrm{p}<0.05)$. MN increased GP skin permeability for the hydrophilic HCL salt of NTX by 10fold and decreased lag time by 10 -fold too. Similar results were found using human skin, such that skin permeability to $\mathrm{NTX} \cdot \mathrm{HCl}$ was elevated to $7.0 \times 10^{-5} \mathrm{~cm} / \mathrm{h}$. Permeability of the primarily unionized (unprotonated) form of NTXOL at pH 8.5 was increased by MN only 3 fold and lag time was only modestly reduced. However, MN treatment with the primarily ionized (protonated) form of NTXOL at pH 4.5 increased skin permeability 5 fold and decreased lag time 4 fold.
\end{abstract}

Conclusion-Enhancement was observed in vitro in both GP and human skin treated with MN compared to intact skin with the salt form of NTX and the ionized form of NTXOL. We conclude that transdermal flux can be optimized by using MN in combination with charged (protonated) drugs that have increased solubility in an aqueous patch reservoir and increased permeability through aqueous pathways created by MN in the skin.

\section{Keywords}

Microneedle; Naltrexone; 6- $\beta$-naltrexol; Transdermal; Protonation 


\section{Introduction}

The treatment of those affected by alcohol and drug abuse is a high priority. It was estimated by Mark et al. that the cost for mental health and drug abuse treatment grew from $\$ 60$ billion in 1991 to $\$ 104$ billion in 2001 (1). In a 2006 survey of American households, 50.9\% of people 12 years of age or older reported that they were currently alcohol drinkers, which translated to 126 million individuals(2,3). Of this population, approximately 22.6 million Americans (or 9.2 $\%$ of the U.S. population) aged 12 or older needed treatment for substance (alcohol or illicit drug) abuse(2). Out of 22.6 million Americans, only 4.0 million people affected received substance abuse treatment $(2,3)$. The latest estimate for the costs to society of illicit drug abuse alone is $\$ 181$ billion (4). At first glance this population treatment number may not seem too high, but it is much more prevalent than the estimated 1.4 million new cases of all cancers projected in 2007(5). At such high costs to society, efforts must be coordinated to provide addicts with safe and effective therapies. Current therapeutics available to patients who suffer from addiction include methadone and buprenorphine treatment, $\mathrm{ReVia}^{\circledR}$, an FDA-approved $50 \mathrm{mg}$ tablet of naltrexone hydrochloride, and the recently FDA-approved 28-day controlled release $380 \mathrm{mg}$ depot form of naltrexone, Vivitrol ${ }^{\mathrm{TM}}(6,7)$. Methadone treatment requires expert supervision in a clinical setting and $\mathrm{ReVia}^{\circledR}$ is poorly bioavailable, with documented side effects (6). Long lasting naltrexone depot formulations have shown effective plasma levels for up to 30 days (8). However, once Vivitrol ${ }^{\mathrm{TM}}$ is injected, it cannot be easily discontinued without painful surgical removal. NTX therapy may need to be discontinued, for example, if the patient requires emergency opiate treatment for pain. Therefore, there is a need for an alternative dosage form of NTX, in order to have an outpatient therapy option that provides controlled release, reduced side effects, and the ability to discontinue therapy easily.

The transdermal route of drug delivery using a drug patch can provide these desirable features for NTX delivery. However, since the SC favors diffusion of lipophilic molecules, conventional transdermal delivery based on passive diffusion often relies on increasing drug hydrophobicity to achieve higher drug permeation in the skin. Guided by this knowledge, previous studies have been carried out in an attempt to increase the hydrophobicity of naltrexone (NTX) by developing prodrugs that would favor passive permeation enhancement (9-11). In these studies (9-11), the more-hydrophobic 3-O-alkyl ester prodrugs of NTX showed significant permeation enhancement over NTX. Despite the increased flux values, permeation levels were still just within the lower limit of a flux range desired for clinical applications, suggesting the need to enhance skin permeability to develop a therapeutic NTX skin patch.

Considering additional strategies to increase NTX delivery across skin, there are a host of physical skin permeability enhancement methods available, such as electroporation, sonophoresis, iontophoresis, and microneedle delivery (12). Microneedle (MN) systems were first conceptualized in the early 1970's to bypass the stratum corneum (SC)(13), but technological limitations at that time prevented product concepts from coming to fruition. Since the microelectronics revolution over the past few decades, technology in micromachining has advanced, allowing great strides in the production of MN and MN arrays. Currently microneedles are made from stainless steel, biodegradable polymers, and silicon $(14,15)$. Likewise, a broad range of compounds such as insulin, desmopressin, oligonucleotides, and vaccines have been screened for permeation enhancement $(14,16-20)$. MNs have been mostly used either as a pretreatment to permeabilize skin before applying a patch or with drug coated onto the MN for rapid dissolution and release in the skin $(14,18,19)$. Data from several studies show promising results, indicating an alternative method for delivering both small and large molecules alike, as compared to standard oral and injectable routes $(14,16-20)$. Kaushik et al. (21)studied the effects of pain associated with $\mathrm{MN}$ insertion in human volunteers and showed that the sensation was no more than that of a smooth surface applied to the skin, or the "sensation of a piece of tape" applied to the skin. Ultimately the goal of microneedle based transdermal 
drug delivery is to deliver pharmacologically active compounds in a pain free manner, by bypassing the $\mathrm{SC}$ or creating aqueous micro-channels across the SC.

To achieve the long term objective of transdermal drug delivery of NTX or its prodrugs via a convenient transdermal drug patch, the goals of this study are to (1) assess the ability of MN to increase NTX delivery across the skin, perhaps to clinically useful levels and (2) investigate future strategies to design prodrugs specifically suitable for delivery across MN-treated skin. This latter goal was studied in the context of the following analysis. The maximum dose of drug that can be delivered across the skin depends on drug solubility in the delivery vehicle (the concentration of the drug applied on the skin surface) and skin permeability to the drug. Both solubility and permeability depend on the structure and charge state of the drug. In conventional transdermal delivery, there is a trade off, where more hydrophilic and charged drugs have greater solubility in aqueous vehicles, but generally smaller permeability across the lipophilic skin barrier. Conversely, more lipophilic and uncharged drugs generally have greater permeability, but smaller aqueous solubility. Aqueous solubility of the drug is desirable to enable the drug to partition into the interstitial fluid once it has permeated across the lipophilic SC barrier for eventual diffusion into the systemic circulation. These competing requirements of having both lipophilic and hydrophilic characteristics in a drug molecule can limit the applicability of conventional transdermal drug delivery.

This study seeks to test the hypothesis that pretreatment of the skin with microneedles to create hydrophilic aqueous micro-channels in the skin's lipophilic barrier can eliminate this trade off, such that hydrophilic and charged drugs will be able to cross the SC through the aqueous channels to yield greater drug fluxes as compared to conventional lipophilic drug molecules. Thus, unlike conventional prodrug design that typically seeks to increase drug lipophilicity, we propose that prodrugs designed for delivery across MN-treated skin should seek to increase drug hydrophilicity instead.

This hypothesis was tested by measuring transdermal flux and permeability of (1) NTX base (lipophilic state, $\mathrm{pK}_{\mathrm{A}}=7.5, \mathrm{cLog} \mathrm{P}=1.92$ ) compared to its charged $\mathrm{HCl}$ salt form (hydrophilic state), (2) of NTXOL ( $\mathrm{pK}_{\mathrm{A}}=7.4, \operatorname{cog} \mathrm{P}=1.32$ ) an alcohol derivative of NTX at two different $\mathrm{pH}$ values of 4.5 and 8.5 , and (3) of NTX base at a low concentration (dose) at pH values of 4.5 and 8.5 , which altered their charge state to create hydrophilic and relatively lipophilic molecules, respectively.

\section{Materials and methods}

\section{Materials}

NTX base was purchased from Mallinckrodt Inc (St. Louis, MO, USA). NTXOL free base and NTX hydrochloride salt were synthesized in the laboratory as described in the procedures below. Hanks' balanced salts modified powder, sodium bicarbonate, and propylene glycol (PG) were purchased from Sigma Chemical (St. Louis, MO, USA). 4-(2-Hydroxyethyl)-1piperazineethanesulfonic acid (HEPES), gentamicin sulfate, trifluoroacetic acid (TFA), triethylamine (TEA), methanol, acetonitrile ( $\mathrm{ACN})$, isopropanol, hydrochloric acid $(\mathrm{HCl})$, and sodium hydroxide were obtained from Fisher Scientific (Fairlawn, NJ, USA). 1-octane sulfonic acid, sodium salt was purchased from ChromTech (Apple Valley, MN, USA).

\section{Microneedle fabrication}

In-plane microneedle rows with five microneedles were cut from stainless steel sheets (Trinity Brand Industries, SS 304, 75 mm thick; McMaster-Carr, Atlanta, GA, USA) using an infrared laser (Resonetics Maestro, Nashua, NH, USA) using methods previously described (19). Briefly, the microneedle row was first drafted in AutoCAD software (Autodesk, Cupertino, 
CA, USA). Using this design, the infrared laser cut microneedles into the stainless steel sheet. The microneedle rows were then cleaned with detergent (Alconox, White Plains, NY, USA) to de-grease the surface and remove part of the slag and oxides deposited during laser-cutting. To completely clean the slag and debris and to sharpen microneedle tips, microneedle rows were electropolished in a solution containing glycerin, ortho-phosphoric acid (85\%) and water in a ratio of 6:3:1 by volume (all chemicals, Fisher Scientific, Fair Lawn, NJ, USA). Electropolishing was performed in a $300 \mathrm{ml}$ glass beaker at $70^{\circ} \mathrm{C}$, a stirring rate of $150 \mathrm{rpm}$, with a current density of $1.8 \mathrm{~mA} / \mathrm{mm}^{2}$ applied for $15 \mathrm{~min}$. A copper plate was used as the cathode (negative), while microneedles acted as the anode (positive). The electropolished microneedle rows were then cleaned by alternatively dipping in $25 \%$ nitric acid (Fisher Scientific) and deionized water with a total of three repetitions. A final rinse was performed under running deionized water before drying under pressurized air. Dry microneedle rows were stored in air-tight containers until later use.

\section{Synthesis procedures}

Naltrexone $\mathrm{HCl}-\mathrm{NTX}$ base $(600 \mathrm{mg}, 1.75 \mathrm{mmol})$ was dissolved in an excess of ACN and cooled in an ice bath. $\mathrm{HCl}(0.053 \mathrm{~mL}, 1.75 \mathrm{mmol})$ in $\mathrm{ACN}$ was added dropwise and the mixture was stirred for $30 \mathrm{~min}$. After the reaction was complete, the solution was evaporated on a rotary evaporator yielding $520 \mathrm{mg} \mathrm{NTX} \cdot \mathrm{HCl}$ (Büchi, Flawil, Switzerland). The salt was freely soluble in water with a melting point of $222-227^{\circ} \mathrm{C}$.

6- $\beta$-Naltrexol base-The synthesis procedure was performed with minor modifications from earlier publications by de Costa et al. as described in Paudel et al.(22,23). $100 \mathrm{~mL}$ of $0.533 \mathrm{M}$ aqueous $\mathrm{NaOH} 13.6 \mathrm{~g}(40.0 \mathrm{mmol})$ was added to NTX free base in an aqueous suspension under argon The alkaline solution of NTX was treated dropwise at ambient temperature during $20 \mathrm{~min}$ with $17.4 \mathrm{~g}(80 \mathrm{mmol})$ of formamidinesulfinic acid dissolved in $200 \mathrm{~mL}$ of $0.533 \mathrm{M}$ aqueous $\mathrm{NaOH}$ (22). After the addition was complete, the solution was heated and stirred at $80-85^{\circ} \mathrm{C}$ for $1.5 \mathrm{~h}$ when thin layer chromatography analysis indicated the reaction to be complete. The reaction mixture was cooled (ice bath) and then treated dropwise under argon with a solution of ammonium chloride $(10.27 \mathrm{~g}, 192 \mathrm{mmol})$ in distilled water (100 $\mathrm{mL}$ ). The aqueous mixture was extracted with $5 \times 100 \mathrm{~mL}$ of $\mathrm{CHCl}_{3}$, and the combined organic extract was filtered through a pad of $\mathrm{Na}_{2} \mathrm{SO}_{4}$ and evaporated in vacuo to afford the crude product (free base) as a foam, which was dissolved in $20 \mathrm{~mL}$ of warm $\left(50^{\circ} \mathrm{C}\right)$ ethyl acetate and diluted to $60 \mathrm{~mL}$ with warm n-hexane (22). Crystallization occurred spontaneously on cooling (22). The crystals were collected by filtration, washed with $2 \times 10 \mathrm{~mL}$ of cold ethyl acetate/nhexane (1:3), and oven-dried in vacuo at $60^{\circ} \mathrm{C}$ to give NTXOL: ${ }^{1} \mathrm{H}$ NMR $\left(\mathrm{CDC1}_{3}\right) 6.71(\mathrm{~d}, \mathrm{~J}$ $=8.1 \mathrm{~Hz}, 1 \mathrm{H}), 6.56(\mathrm{~d}, \mathrm{~J}=8.1 \mathrm{~Hz}, 1 \mathrm{H}), 4.55(\mathrm{~d}, \mathrm{~J}=6.1 \mathrm{~Hz}, 1 \mathrm{H}), 3.57(\mathrm{~m}, 1 \mathrm{H})$.

Solubility of NTX.HCl and NTXOL Base at pH 4.5 and $8.5-\mathrm{An}$ excess of NTX $\cdot \mathrm{HCl}$ $(\sim 350 \mathrm{mg})$ was added to $1 \mathrm{~mL}$ of 3:1 PG:Hanks' buffer $\mathrm{pH}$ 7.4. The saturated solution was then vortexed for $1 \mathrm{~min}$, sonicated for $15 \mathrm{~min}$, and placed in an incubator at $32^{\circ} \mathrm{C}$ for $24 \mathrm{~h}$ while shaking on a belly shaker. Just before filtering, a glass syringe and $0.45 \mu \mathrm{m}$ filter was heated in an incubator to $32^{\circ} \mathrm{C}$. The solution was filtered, and appropriate dilution with ACN was completed for high pressure liquid chromatography (HPLC) analysis for NTX content. Solubility was determined in triplicate.

The solubility of naltrexol base at $\mathrm{pH} 4.5$ was determined by adding an excess of NTXOL base to the solvent and adjusting the $\mathrm{pH}$ to 4.5 with $5 \mathrm{M} \mathrm{HCl}$, and then separating out the excess solid by centrifugation. Samples were analyzed for NTXOL concentration by HPLC. The solubility of naltrexol base at $\mathrm{pH} 8.5$ was determined by the same method, except the solution was $\mathrm{pH}$ adjusted to 8.5 with $3 \mathrm{~N} \mathrm{NaOH}$. 


\section{Formulation preparation}

NTX base vs. NTX $\cdot \mathbf{H C l}-A$ saturated solution of NTX base $(100 \mathrm{mg})$ was prepared in $3: 1$ PG:Hanks' buffer $\mathrm{pH} 7.4$ and the mixture was sonicated for $10 \mathrm{~min}$ and then applied onto the skin. The final $\mathrm{pH}$ of this solution was approximately 7.6. A base equivalent amount of $\mathrm{NTX} \cdot \mathrm{HCl}(111.00 \mathrm{mg}$, which corresponds to $100 \mathrm{mg}$ NTX and $11 \mathrm{mg} \mathrm{HCl})$ was dissolved in 3:1 PG:Hanks' buffer pH 7.4 by sonication for $10 \mathrm{~min}$, which had a final $\mathrm{pH}$ value of 4.9.

NTXOL base pH 8.5 vs. NTXOL base pH 4.5-100 mg of NTXOL free base was prepared

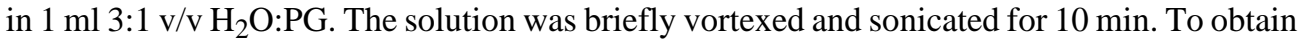
a mixture of $\mathrm{pH} 8.5 \mathrm{NTXOL}$ solution, $0.5 \mathrm{~N}$ sodium hydroxide was added. The same procedure was performed to obtain a $\mathrm{pH} 4.5$ solution, except that $\mathrm{pH}$ adjustment was carried out by adding $0.5 \mathrm{M} \mathrm{HCl}$ dropwise. The ionization of NTXOL free base upon $\mathrm{pH}$ adjustment by $\mathrm{HCl}$ addition resulted in complete dissolution to form a solution of $292.4 \mathrm{mM}$. The identical drug concentration solutions used for comparison at $\mathrm{pH} 8.5$ were saturated with excess solid (solubility $2.69 \mathrm{mM}$ ). The tertiary amine in NTXOL has a pKa of 7.4 (24).

NTX base pH 8.5 vs. NTX base pH 4.5-20 mg of NTX free base was prepared in $3 \mathrm{ml}$ $3: 1 \mathrm{v} / \mathrm{v}_{2} \mathrm{O}: \mathrm{PG}$ in duplicate. The solution was briefly vortexed and sonicated for 10 minutes, resulting in a saturated solution. To obtain equal concentrations of NTX at pH values of 4.5 and 8.5 , both $3 \mathrm{ml}$ aliquots were adjusted to $\mathrm{pH} 8.5$ with $.5 \mathrm{M} \mathrm{HCl}$. Both saturated preparations were then filtered through a Pall Acrodisc ${ }^{\circledR}$ Premium $25 \mathrm{~mm}$ syringe filter $(0.45 \mu \mathrm{m}$ nylon membrane), resulting in a clear formulation at the solubility limit $(14.5 \mathrm{mM})$ at $\mathrm{pH} 8.5$ in $3: 1$ $\mathrm{H}_{2} \mathrm{O}: \mathrm{PG}$. One of the two equimolar solutions was then $\mathrm{pH}$ adjusted with $0.5 \mathrm{M} \mathrm{HCl}$ to $\mathrm{pH} 4.5$ allowing for almost complete ionization of the tertiary amine in NTX.

Visualizing micro-pores created by microneedle insertion-To determine the ability of microneedle rows to penetrate the skin and create micro-pores, microneedle rows $(n=3)$ were inserted into human skin, and gentian violet (a violet topical antifungal agent, $2 \%$ solution, Humco, Texarkana, TX, USA) was then applied on the treated site for $1 \mathrm{~min}$ and wiped away using isopropanol swabs. Gentian violet selectively stained the sites of skin perforation, which identified the sites of microneedle insertion. The use of human skin was approved for these experiments by the Georgia Tech IRB.

In vitro skin diffusion studies-Hairless guinea pig skin was harvested from euthanized animals. Animal studies were approved by the University of Kentucky IACUC. Human skin harvested during abdominoplasty was used for the diffusion studies. Human tissue use was approved by the University of Kentucky Institutional Review Board. Full thickness skin (approx. $1 \mathrm{~mm}$ thick) sections were obtained by removing the subcutaneous fatty tissue by scalpel dissection, and were stored at $-20^{\circ} \mathrm{C}$. A PermeGear flow-through (In-Line, Riegelsville, PA, USA) diffusion cell system was used for the skin permeation studies. Skin used for microneedle treatment was placed on a wafer of polydimethylsiloxane polymer, which mimicked the underlying mechanical support of tissue because of its comparable structural flexibility and elasticity. The skin was pierced 20 times with an array containing $5 \mathrm{MN}$ (i.e., to make a total of 100 individual and non-over lapping piercings) before mounting the skin in the diffusion cell. Single MN sections of 5 needles for practical experimental design purposes were used. If any damage to an $\mathrm{MN}$ section was observed the section was replaced. Cells containing MN treated skin showed no presence of receiver fluid back flow in the donor compartment. Untreated skin samples were simply placed in the diffusion cells. Diffusion cells were kept at $32^{\circ} \mathrm{C}$ using a circulating water bath. Data were collected using skin from a single guinea pig or human donor with three cells for untreated formulations and 3-4 cells for the MN treated formulations $(n=9-12)$. The experiments were performed 3 times with three separate piece of skin $(\mathrm{N}=3)$. The physiological receiver solution was HEPES-buffered Hanks' balanced 
salts with gentamicin $(0.1 \mathrm{mM})$ at $\mathrm{pH} 7.4(25)$, and the flow rate was adjusted to $1.1 \mathrm{~mL} / \mathrm{h}$. Each cell was charged with $0.25 \mathrm{~mL}$ of the respective drug solution in the donor compartment of the chamber. Samples were collected from the receiver compartment in six-hour increments over $48 \mathrm{~h}$. All samples were stored at $4^{\circ} \mathrm{C}$ until processed by solid phase extraction. The cumulative quantity of drug collected in the receiver compartment was plotted as a function of time. The flux value for a given experiment was obtained from the slope of the steady state portion of the cumulative amount of drug permeated, plotted over time. Apparent permeability coefficient values were calculated from Fick's First Law of diffusion:

$$
\frac{1}{A}\left(\frac{d M}{d t}\right)=J_{s}=K_{p} \Delta C
$$

(Equation 1)

where $J_{S}$ is the flux at steady state $\left(\mathrm{nmol} / \mathrm{cm}^{2} / \mathrm{h}\right), M$ is the cumulative amount of drug permeating through the skin, $t$ is time, $A$ is the area of the skin $\left(0.95 \mathrm{~cm}^{2}\right), K_{p}$ is the effective permeability coefficient in $\mathrm{cm} / \mathrm{h}$, and $\Delta C$ is the difference in concentrations of NTX or NTXOL in the donor and receiver components. Sink conditions were maintained in the receiver solution for the duration of the experiment; thus $\Delta C$ was approximated by the initial drug concentration in the donor compartment.

In the context of data analysis, theoretical dosing was computed from Eq. 2,

$$
\mathrm{X}=\mathrm{A}_{\mathrm{p}} \mathrm{K}_{\mathrm{p}} \mathrm{S}
$$

where $\mathrm{X}$ is the theoretical dosing rate $(\mathrm{mg} / \mathrm{h}), \mathrm{A}_{\mathrm{p}}$ is the area of the applied patch (in $\mathrm{cm}^{2}$ ), $\mathrm{K}_{\mathrm{p}}$ is the permeability of $\mathrm{NTX} \cdot \mathrm{HCl}$ in microneedle-treated human skin (e.g. $7.0 \times 10^{-5} \mathrm{~cm} / \mathrm{h}$, as determined in the Results section) and S is the solubility (e.g. NTX $\cdot \mathrm{HCl}=300 \mathrm{mg} / \mathrm{cm}^{3}$ ).

Quantitative analysis-Quantitative analysis of NTX concentrations by HPLC was carried out using a modification of the assay described by Hussain et al.(26). The HPLC analysis was performed as reported by Vaddi et al. (27). The HPLC system consisted of a Waters 717plus autosampler, a Waters 1525 Binary HPLC pump, and a Waters 2487 dual wavelength absorbance detector with Waters Breeze ${ }^{\mathrm{TM}}$ software (Milford, Massachusetts, USA). A Brownlee (Wellesley, Massachusetts, USA) C-18 reversed-phase Spheri-5 $\mu$ m column $(220 \times$ $4.6 \mathrm{~mm})$ with a C-18 reversed phase $7 \mu \mathrm{m}$ guard column $(15 \times 3.2 \mathrm{~mm})$ by Perkin Elmer ${ }^{\circledR}$ was used with the UV detector set at a wavelength of $215 \mathrm{~nm}$. The mobile phase for NTX was 70:30 ACN: $0.1 \%$ TFA with $0.065 \%$ 1-octane sulfonic acid sodium salt, adjusted to $\mathrm{pH} 3.0$ with TEA, and samples were run at a flow rate of $1.5 \mathrm{~mL} / \mathrm{min}$ with a run time of $5 \mathrm{~min}$. The mobile phase for NTXOL consisted of 90:10 ACN:0.1\% TFA adjusted to $\mathrm{pH} 3.0$ with TEA. Samples were run for $10 \mathrm{~min}$ at $1 \mathrm{~mL} / \mathrm{min}$ as described by Paudel et al. (23). For both NTX and NTXOL, a one hundred microliter sample of analyte was injected onto the column. Samples were analyzed within a linear range of the standard curves from $100-25000 \mathrm{ng} / \mathrm{mL}$. NTX and NTXOL exhibited excellent linearity over the entire concentration range employed in the assays.

NTX and NTXOL were extracted from the buffer samples by solid phase extraction (30mg 1cc Oasis ${ }^{\circledR}$ HLB, Waters Corp., Milford, MA, USA) as described by Vaddi et al.(27). Before extracting the aqueous drug samples $(5 \mathrm{~mL})$, the solid phase cartridge was conditioned with 1 $\mathrm{mL}$ of methanol, followed by $1 \mathrm{~mL}$ of nanopure water. After sample loading, the cartridge was washed with $1 \mathrm{~mL}$ of $5 \%$ aqueous methanol and the drug was eluted with $1.1 \mathrm{~mL} \mathrm{ACN}$ and analyzed by HPLC. A similar extraction method was employed for NTXOL, except $2 \mathrm{~mL}$ of methanol was used in the cartridge conditioning, and $1 \mathrm{~mL}$ of nanopure water was used to further wash the samples after being loaded onto the cartridge. NTXOL was eluted with $2 \mathrm{~mL}$ 
of isopropanol and finally with $1 \mathrm{~mL}$ ACN. NTXOL samples were evaporated under nitrogen in a water bath maintained at $37^{\circ} \mathrm{C}$. The samples were then reconstituted with $1 \mathrm{~mL}$ of ACN for HPLC analysis. Highly concentrated (outside of the standard curve range) samples of NTX and NTXOL were further diluted 10 times with ACN.

In vitro data analysis-The data obtained from the diffusion experiments were plotted as the cumulative amount of drug collected in the receiver compartment as a function of time. Results for NTX base and NTX $\cdot \mathrm{HCl}$, and for NTXOL base adjusted to $\mathrm{pH} 4.5$ and 8.5 in GP skin were each compiled from skin from three different sacrificed animals $(\mathrm{N}=3, \mathrm{n}=9-12)$. The $100 \mathrm{mg} / \mathrm{mL}$ study of $\mathrm{NTX} \cdot \mathrm{HCl}$ in human skin was compiled from one piece of skin $(\mathrm{n}=3)$. The statistical analysis of data was computed with Student's t-test and one-way ANOVA with posthoc Tukey's pairwise test using SIGMA-STAT (SPSS, Inc., Chicago, IL, USA) software.

\section{Results and discussion}

\section{Naltrexone and naltrexol solubilities}

Transdermal delivery was studied for NTX and NTXOL with different charge states, which affected skin permeability and solubility in the delivery vehicle. The structures of the compounds used in this study are shown in Fig. 1. As illustrated in Fig. 1, the hydrochloride is formed by attachment to NTX free base (1a.) at the tertiary amine (1b.). Similarly, addition of $0.5 \mathrm{M} \mathrm{HCl}$ to NTXOL resulted in protonation of the tertiary amine (1d.) whereas addition of $0.5 \mathrm{~N} \mathrm{NaOH}$ to NTXOL free base resulted in the compound remaining primarily in the unprotonated form (1c.). Addition of $\mathrm{HCl}$ to NTXOL enhanced the solubility by protonation of the tertiary amine at $\mathrm{pH} 4.5$ forming a clear solution, whereas solubility was not enhanced by $\mathrm{NaOH}$ addition.

Solubility values of NTX can be observed in Table 1. By converting the base form of NTX to the more soluble hydrochloride salt $(\mathrm{NTX}-\mathrm{HCl})$, the solubility was increased by 18.2 times in 3:1 PG:buffer. Solubilities of NTXOL free base in $3: 1 \mathrm{H}_{2} \mathrm{O}: \mathrm{PG}$ were determined at $\mathrm{pH} 8.5$ and $\mathrm{pH} 4.5$ (Table 2). The $\mathrm{pH} 4.5$ solutions were completely solubilized at the experimental concentrations employed for MN/ionization analysis, as shown in Table 2. Thus, the solubility of the drugs in the solvent systems used in these studies is ranked NTXOL $(\mathrm{pH} 4.5)>\mathrm{NTX} \cdot \mathrm{HCl}$ $>$ NTX base > NTXOL (pH 8.5).

\section{Microneedle rows create micro-pores in the skin}

The microneedle rows used in the permeation study had five 750 micron long microneedles (Fig. 2A and 2B). To visually examine the microneedle penetration sites of the rows, the skin surface was imaged after microneedle insertion and gentian violet treatment. Gentian violet was wiped off from the skin surface using isopropanol swabs, but remained within the pores created by the microneedles, to stain the sites of microneedle penetration (Fig. 2C). Once microneedles were applied to the skin, they appeared to be fully inserted up to the base of the row, similar to results shown by Martanto et al. (19).

\section{In vitro permeation studies of NTX in human and guinea pig skin}

Skin permeation studies of NTX base (lipophilic state) and NTX $\bullet \mathrm{HCl}$ salt (hydrophilic state) with and without the use of MNs provided insight on the effects of solubility on permeation via aqueous pathways created by MN treatment. The in vitro permeation profiles from NTX free base and NTX hydrochloride salt across GP with and without MN pretreatment can be observed in Fig. 3a. Fig. 3b shows the comparison of MN or no-MN permeation of NTX $\cdot \mathrm{HCl}$ across human skin. 
Considering intact skin (i.e., without MN treatment), NTX base was delivered across skin much more effectively than the $\mathrm{NTX} \cdot \mathrm{HCl}$ (Fig. 3a). This is expected, because the skin barrier should be more permeable to the lipophilic NTX base than the hydrophilic NTX $\bullet \mathrm{HCl}$. More specifically, the steady state flux of NTX base $\left(9.94 \pm 2.75 \mathrm{nmol} / \mathrm{cm}^{2} / \mathrm{h}\right)$ was greater than $\mathrm{NTX} \cdot \mathrm{HCl}\left(2.71 \pm 1.20 \mathrm{nmol} / \mathrm{cm}^{2} / \mathrm{h}\right)(\mathrm{p}<0.05)$ and the lag time of NTX base $(21.22 \pm 3.91 \mathrm{~h})$ was statistically the same as $\mathrm{NTX} \cdot \mathrm{HCl}(22.38 \pm 7.68 \mathrm{~h})(\mathrm{p}>0.05)$ (Table 1).

After treatment with MN, the reverse trend was observed. In this case, NTX $\cdot \mathrm{HCl}$ was delivered across skin much more efficiently than NTX base (Fig. 3a). This is consistent with the hypothesis that $\mathrm{MN}$ treatment makes aqueous micro-channels into the skin, which favors transport of hydrophilic molecules across the skin. More specifically, the steady state flux of $\mathrm{NTX} \cdot \mathrm{HCl}\left(27.16 \pm 4.57 \mathrm{nmol} / \mathrm{cm}^{2} / \mathrm{h}\right)$ was greater than NTX base $\left(11.80 \pm 5.02 \mathrm{nmol} / \mathrm{cm}^{2} / \mathrm{h}\right)$ $(\mathrm{p}<0.05)$ and the lag time of NTX $\cdot \mathrm{HCl}(2.41 \pm 3.85 \mathrm{~h})$ was shorter than NTX base $(18.91 \pm$ $4.49 \mathrm{~h})(\mathrm{p}<0.05)($ Table 1$)$.

It is interesting to note that there was no significant difference $(\mathrm{p}>0.05)$ in steady state fluxes of NTX base between MN-treated $\left(11.80 \pm 5.02 \mathrm{nmol} / \mathrm{cm}^{2} / \mathrm{h}\right)$ and intact $(9.94 \pm 2.75 \mathrm{nmol} /$ $\mathrm{cm}^{2} / \mathrm{h}$ ) skin (Table 1). Likewise, the lag times remained unchanged upon comparison of $\mathrm{MN}$ $(18.91 \pm 4.49 \mathrm{~h})$ and intact $(21.22 \pm 3.91 \mathrm{~h})$ GP skin $(\mathrm{p}>0.05)$. Together, these observations indicate that the transdermal pathway taken by NTX base remained essentially the same (the permeability values are similar), even when the SC had been pierced by the MN. Due to the low aqueous solubility of NTX base, its molecules were unable to utilize the alternate pathway created by microneedles across the $\mathrm{SC}$ in the form of aqueous micro-channels.

In contrast, significant enhancement $(\mathrm{p}<0.05)$ was observed when comparing the fluxes of $\mathrm{NTX} \cdot \mathrm{HCl}$ across $\mathrm{MN}$-treated and intact GP skin (Table 1). As expected, under normal passive diffusion conditions a more ionized, water-soluble compound permeates the skin at a slower rate (the permeability values are higher for the primarily unprotonated drugs in intact skin). Conversely, when the lipid bilayer barrier is by-passed by an MN-created aqueous channel, significant enhancement in drug permeation is observed, thereby supporting the fact that an alternate mechanism of permeation occurs in the presence of MN-treatment. Further support of this hypothesis is demonstrated by the significant change in lag time that occurs when GP skin is treated with MN. When untreated, a lag time for NTX $\cdot \mathrm{HCl}$ of $22.38 \pm 7.68 \mathrm{~h}$ was observed, compared to $2.41 \pm 3.85 \mathrm{~h}$ for MN-treated GP skin ( $\mathrm{p}<0.05$ ). The observation of a decreased time to reach steady state demonstrates the presence of an alternative permeation pathway.

Upon comparison of GP skin to human skin, flux levels were lower in human skin (Fig 3b), as observed in a previous study (28). However, in the NTX $\cdot \mathrm{HCl}$ experiment a similar trend in the $\mathrm{MN}$-treatment enhancement level was observed in both types of skin, i.e. 8-fold flux enhancement $(\mathrm{p}<0.05)$ in human skin compared to a 10 -fold flux enhancement in GP skin. Significant decreases $(\mathrm{p}<0.05)$ in lag times were also observed with MN-treatment in both tissue types. This initial finding suggests that either human or GP skin can be used with confidence in future in vitro studies. It was also necessary to obtain the human skin flux values in order to determine if the $\mathrm{MN}$ treatment would allow therapeutic doses of drug to cross human skin at an adequate rate for clinical scenarios. Equation 2 reveals that NTX free base delivered by passive diffusion without $\mathrm{MN}$ should deliver a maximum daily dose of $0.4 \mathrm{mg}$ for a 10 $\mathrm{cm}^{2}$ patch containing NTX free base at its solubility limit $(43.6 \mathrm{mM})$, and a permeability of $1.08 \times 10^{-4} \mathrm{~cm} / \mathrm{h}$ in human skin (23). This dose is unlikely to be therapeutic [3]. In contrast, $\mathrm{NTX} \cdot \mathrm{HCl}$ delivery using $\mathrm{MN}$-treated human skin and a $10 \mathrm{~cm}^{2}$ patch should reach a maximum theoretical daily dose of $5.0 \mathrm{mg}$ (calculated from Eq. 2) at the solubility maximum (794.4 mM) using the measured permeability of $7.0 \times 10^{-5} \mathrm{~cm} / \mathrm{h}$. This dose is likely to be in the therapeutic 
range, given that systemic absorption from an oral daily dose ranges from 2.5 to $20 \mathrm{mg}$ for a $50 \mathrm{mg}$ tablet (6).

\section{In vitro NTXOL base ionization guinea pig diffusion studies}

After determining the ability of MNs to enhance permeability and flux of a hydrophilic NTX salt form, next we wanted to devise a strategy of prodrug design that will enable development of more hydrophilic drugs to further enhance the flux. One strategy to develop hydrophilic prodrugs can involve increasing the ionization (protonation in this case) of the molecule. We selected NTXOL, NTX's more polar active metabolite, for these flux measurement studies. NTXOL has a tertiary amine, which can be protonated or deprotonated to change the charge on NTXOL. The $\mathrm{pH}$ of the free base solution of NTXOL was adjusted to study the effects of protonation of the tertiary amine group upon skin permeation. At $\mathrm{pH} 4.5 \mathrm{NTXOL}$ has a net positive charge, while at $\mathrm{pH} 8.5$ the amine group is more than 90 percent unionized. It was our hypothesis that upon ionization (protonation) of NTXOL at $\mathrm{pH} 4.5$ with $\mathrm{HCl}, \mathrm{MN}$ application should enhance permeation through GP skin because of the aqueous solubility enhancement. As shown in Fig. 4a, there is a statistically significant increase $(\mathrm{p}<0.05)$ in the NTXOL flux at $\mathrm{pH} 8.5$ in MN-treated and MN-untreated GP skin. However, these $\mathrm{pH} 8.5$ flux values are similar in magnitude to the NTXOL flux at pH 4.5 in MN-untreated GP skin, and all of these flux values are more than five-fold less than the NTXOL flux at $\mathrm{pH} 4.5$ (protonated) in MN-treated GP skin. Thus, at pH 8.5, as more of the NTXOL is in the unprotonated form, the NTXOL solubility in the predominantly aqueous formulation is low, resulting in low flux levels. As expected, analysis of variance of the $\mathrm{pH} 4.5 \mathrm{MN}$-untreated skin compared to $\mathrm{pH} 8.5 \mathrm{MN}$-treated and MN-untreated skin (Fig. 4a) and the base form of NTX revealed no significant enhancement ( $\mathrm{p}>0.05)$. At $\mathrm{pH} 4.5$, NTXOL is protonated and has high aqueous solubility and therefore does not permeate efficiently through intact SC lipid bilayers. However, significant enhancement of MN-treated GP skin dosed with NTXOL at pH 4.5 was observed over NTXOL at $\mathrm{pH} 8.5$ as shown in Fig. $4 \mathrm{~b}(\mathrm{p}<0.05)$. Significant enhancement in flux was also observed when comparing MN treated skin dosed with NTXOL pH 4.5 to untreated skin dosed with NTXOL pH 4.5 and $\mathrm{pH}$ 8.5. A comparison of flux values of MN-treated GP skin dosed with NTXOL pH 4.5 and NTX $\bullet H C l$ showed a slight 1.3 -fold enhancement for NTXOL, although the permeability values are similar. The two drugs are structurally very similar, and the difference in flux can also be explained by the higher solubility of protonated NTXOL over $\mathrm{NTX} \cdot \mathrm{HCl}$.

Comparing a previous experiment (unpublished) to the current study demonstrated that $\mathrm{MN}$ treated GP skin had the same permeation for NTXOL $\cdot \mathrm{HCl}\left(36.12 \pm 1.77 \mathrm{nmol} / \mathrm{cm}^{2} / \mathrm{h}\right)$ and NTXOL at pH $4.5\left(35.07 \pm 8.18 \mathrm{nmol} / \mathrm{cm}^{2} / \mathrm{h}\right)$, whereas MN-untreated skin showed different flux values for NTXOL $\cdot \mathrm{HCl}\left(2.75 \pm 1.38 \mathrm{nmol} / \mathrm{cm}^{2} / \mathrm{h}\right)$ and NTXOL at pH $4.5(6.56 \pm 6.49$ $\mathrm{nmol} / \mathrm{cm}^{2} / \mathrm{h}$ ). Significant protonation of NTXOL results in slow permeabilities with no MN treatment, as would be expected for a water soluble amine salt crossing through intact SC lipid bilayers. Similarly, NTXOL free base at $\mathrm{pH} 8.5$ has a low aqueous solubility of $2.69 \mathrm{mM}$ in 3:1 $\mathrm{H}_{2} \mathrm{O}: \mathrm{PG}$, and the flux values are also low with the $\mathrm{MN}$ treatment for this compound.

Calculated lag times from NTXOL permeation profiles were consistent with the results obtained with NTX free base and NTX hydrochloride. The NTXOL MN-untreated pH 4.5 and $8.5 \mathrm{lag}$ times were $25.97 \pm 5.50 \mathrm{~h}$ and $24.34 \pm 5.17 \mathrm{~h}$, respectively, while the MN-treated pH 4.5 and 8.5 lag times were $6.58 \pm 5.02 \mathrm{~h}$ and $17.53 \pm 6.56 \mathrm{~h}$, respectively, a significant change with $\mathrm{MN}$ treatment $(\mathrm{p}<0.05)$. This significant decrease in lag time demonstrates that the compound changed its primary pathway of diffusion. Enhanced solubility by protonation provides a means for facile permeation through the $\mathrm{MN}$ created aqueous channels, thereby bypassing the SC. 
As seen in Table 2, similar trends were observed for NTXOL as those observed for NTX (Table 1). The common trend of enhanced flux and reduction in lag time remains consistent for MNtreatments as compared to no $\mathrm{MN}$-treatment. The use of MNs significantly enhanced skin permeability for hydrophilic molecules - NTXOL-pH 4.5 and NTX $\bullet \mathrm{HCl}$, by 6 to 10 fold.

Initially it was theorized that ionization may play a role in the driving force for permeation through aqueous $\mathrm{MN}$-created pores as shown by permeation enhancement of charged NTXOL. Therefore, a low concentration diffusion study was performed. Equimolar concentrations in solution $(14.5 \mathrm{mM})$ of NTX base at pH 4.5 (ionized) and 8.5 (unionized) were applied to GP skin, and results are shown in Table 2 . There is a trend of enhancement in permeation and a decrease in lagtime of the $\mathrm{MN}$ treated skin over untreated skin at both $\mathrm{pH} 4.5$ and 8.5. However, there is no significant difference in permeation between the NTX pH 4.5 and NTX pH 8.5 intact skin ( $\mathrm{p}>0.05$ ) or the $\mathrm{pH} 4.5$ and NTX pH $8.5 \mathrm{MN}$-treated skin ( $\mathrm{p}>0.05)$. Therefore, the driving force for NTX and NTXOL pH 4.5 is not governed by an electronic state, rather the electronic state influences solubility and solubility drives enhanced permeation as indicated by the previously discussed diffusion studies. A high solubility of the hydrophilic molecules is expected to further enhance the flux across the skin. It is expected that in vivo, the hydrophilic drugs will exhibit rapid diffusion (reduced lag times) into the systemic circulation for higher drug bioavailability, as compared to more lipophilic drugs.

\section{Conclusions}

Microneedle-assisted delivery has been shown to be a useful technique in the enhancement of flux for the transdermal drug candidate NTX. Similarly, NTX and NTXOL are excellent molecules for use in the study of $\mathrm{MN}$-assisted transdermal delivery due to their slow diffusivity in their free base forms. Unlike traditional passive transdermal delivery which relies mostly on non-ionized lipid solubilized formulation efforts, MN-assisted delivery relates closer to formulation of injectable drugs in that it is based on total drug solubility. The charged species propagates total drug solubility and thus enhances permeation through the MN-created pores. Modifications to increase the solubility of transdermal candidates by using salts of weak bases instead of the free bases themselves, or utilizing water soluble prodrugs may be an integral part of microneedle drug candidate selection and optimization. Reversal of the permeation pathway through aqueous MN created channels significantly enhanced the flux of the highly water soluble hydrochloride salt form of NTX. MN treatment is useful in improving the flux of $\mathrm{NTX} \cdot \mathrm{HCl}$ because the solubility of NTX is substantially improved in this formulation, even though MN treatment does not increase the permeability of the skin to the protonated NTX salt form over unprotonated NTX base. Flux enhancement of NTX $\bullet \mathrm{HCl}$ to levels in the therapeutic delivery rate range was demonstrated with $\mathrm{MN}$ treatment using human skin in vitro. Further studies are being conducted in order to determine the optimal formulations needed for $\mathrm{MN}$ assisted transdermal drug delivery.

\section{ACKNOWLEDGMENTS}

We would like to thank Dr. Mark Allen at Georgia Tech for the use of his microfabrication facilities. This research was supported in part by NIH R01DA13425. Human skin was supplied by the National Cancer Institute (NCI) Cooperative Human Tissue Network (CHTN). HSG and MRP are members of the Center for Drug Design, Development and Delivery and the Institute for Bioengineering and Bioscience at Georgia Tech. MRP is the Emerson Lewis Faculty Fellow.

\section{References}

1. Mark TL, Coffey RM, Vandivort-Warren R, Harwood HJ, King EC. U.S. spending for mental health and substance abuse treatment, 1991-2001. Health Aff (Millwood) 2005;(Suppl Web Exclusives):W5-133-W135-142. [PubMed: 15797947] 
2. SAMHSA. Results from the 2006 National Survey on Drug Use and Health: National Findings. Department of Health and Human Services: Substance Abuse and Mental Health Services Administration Office of Applied Studies 2006:1-282.

3. Bouwstra JA, Honeywell-Nguyen PL, Gooris GS, Ponec M. Structure of the skin barrier and its modulation by vesicular formulations. Progress in lipid research 2003;42:1-36. [PubMed: 12467638]

4. NIDA. Treatment for Drug Abusersin the Criminal Justice System. National Institutes of Health; National Institiute on Drug Abuse. July;2006

5. ACS. Cancer Facts and Figures 2007. American Cancer Society 2007:1-56.

6. PDR. Medical Economics. New Jersey (Generics second edition) 1996:1083-1086.

7. Alkermes, C. Vivitrol ${ }^{\mathrm{TM}}$ (naltrexone for extended-release injectable suspension). http://www.vivitrol.com/

8. Galloway GP, Koch M, Cello R, Smith DE. Pharmacokinetics, safety, and tolerability of a depot formulation of naltrexone in alcoholics: an open-label trial. BMC Psychiatry 2005;5:1-10. [PubMed: 15631624]

9. Hammell DC, Hamad M, Vaddi HK, Crooks PA, Stinchcomb AL. A duplex “Gemini” prodrug of naltrexone for transdermal delivery. J Control Release 2004;97:283-290. [PubMed: 15196755]

10. Hammell DC, Stolarczyk EI, Klausner M, Hamad MO, Crooks PA, Stinchcomb AL. Bioconversion of naltrexone and its 3-O-alkyl-ester prodrugs in a human skin equivalent. J Pharm Sci 2005;94:828836. [PubMed: 15736197]

11. Nalluri BN, Milligan C, Chen J, Crooks PA, Stinchcomb AL. In vitro release studies on matrix type transdermal drug delivery systems of naltrexone and its acetyl prodrug. Drug Dev Ind Pharm 2005;31:871-877. [PubMed: 16305998]

12. Prausnitz MR, Mitragotri S, Langer R. Current status and future potential of transdermal drug delivery. Nature reviews 2004;3:115-124.

13. Gersteland, MS.; Place, VA. Drug delivery device, Alza Corporation. U.S.P. Office., editor. Vol. 3,964,482. Alza Corporation; USA: 1976. p. 1-13.

14. Prausnitz MR. Microneedles for transdermal drug delivery. Adv Drug Deliv Rev 2004;56:581-587. [PubMed: 15019747]

15. Prausnitz, MR.; Mikszta, JA.; Raeder-Devens, J. Microneedles in Percutaneous Penetration Enhancers. E.S.a.H. Maibach., editor. CRC Press; Boca Raton, FL: 2005. p. 239-255.

16. Coulman SA, Barrow D, Anstey A, Gateley C, Morrissey A, Wilke N, Allender C, Brain K, Birchall JC. Minimally invasive cutaneous delivery of macromolecules and plasmid DNA via microneedles. Curr Drug Deliv 2006;3:65-75. [PubMed: 16472095]

17. Gardeniers JGE, Luttge JW, Berenschott JW, de Boer MJ, Yeshurun Y, Hefetz M, van't Oever R, van den Berg A. Silicon micromachined hollow microneedles for transdermal liquid transport. J MEMS 2003;6:855-862.

18. Cormier M, Johnson B, Ameri M, Nyam K, Libiran L, Zhang DD, Daddona P. Transdermal delivery of desmopressin using a coated microneedle array patch system. J Control Release 2004;97:503511. [PubMed: 15212882]

19. Martanto W, Davis SP, Holiday NR, Wang J, Gill HS, Prausnitz MR. Transdermal delivery of insulin using microneedles in vivo. Pharm Res 2004;21:947-952. [PubMed: 15212158]

20. Mikszta JA, Alarcon JB, Brittingham JM, Sutter DE, Pettis RJ, Harvey NG. Improved genetic immunization via micromechanical disruption of skin-barrier function and targeted epidermal delivery. Nature medicine 2002;8:415-419.

21. Kaushik S, Hord AH, Denson DD, McAllister DV, Smitra S, Allen MG, Prausnitz MR. Lack of pain associated with microfabricated microneedles. Anesth Analg 2001;92:502-504. [PubMed: 11159258]

22. de Costa BR, Iadarola MJ, Rothman RB, Berman KF, George C, Newman AH, Mahboubi A, Jacobson AE, Rice KC. Probes for narcotic receptor mediated phenomena. 18. Epimeric 6 alpha- and 6 betaiodo-3,14-dihydroxy-17-(cyclopropylmethyl)-4,5 alpha-epoxymorphinans as potential ligands for opioid receptor single photon emission computed tomography: synthesis, evaluation, and radiochemistry of [125I]-6 beta-iodo-3,14-dihydroxy-17-(cyclopropylmethyl)-4,5 alphaepoxymorphinan. J Med Chem 1992;35:2826-2835. [PubMed: 1322988] 
23. Paudel KS, Nalluri BN, Hammell DC, Valiveti S, Kiptoo P, Hamad MO, Crooks PA, Stinchcomb AL. Transdermal delivery of naltrexone and its active metabolite 6-beta-naltrexol in human skin in vitro and guinea pigs in vivo. J Pharm Sci 2005;94:1965-1975. [PubMed: 16052561]

24. Liu J, Gong Z. Progress of research on naltrexol. Zhongguo Yaowu Yilaixing Zazhi 2005;14:89-92.

25. Bronaugh RL, Stewart RF. Methods for in vitro percutaneous absorption studies III: hydrophobic compounds. J Pharm Sci 1984;73:1255-1258. [PubMed: 6491945]

26. Hussain MA, Koval CA, Myers MJ, Shami EG, Shefter E. Improvement of the oral bioavailability of naltrexone in dogs: a prodrug approach. J Pharm Sci 1987;76:356-358. [PubMed: 3656096]

27. Vaddi HK, Hamad MO, Chen J, Banks SL, Crooks PA, Stinchcomb AL. Human skin permeation of branched-chain 3-0-alkyl ester and carbonate prodrugs of naltrexone. Pharm Res 2005;22:758-765. [PubMed: 15906171]

28. Valiveti S, Hammell DC, Paudel KS, Hamad MO, Crooks PA, Stinchcomb AL. In vivo evaluation of 3-O-alkyl ester transdermal prodrugs of naltrexone in hairless guinea pigs. J Control Release 2005;102:509-520. [PubMed: 15653167] 


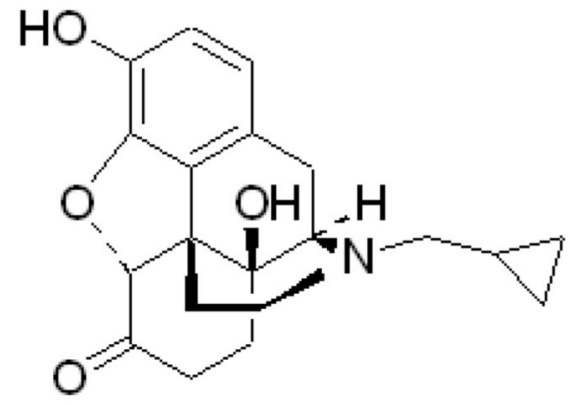

a. Naltrex one free base

MW 341.4

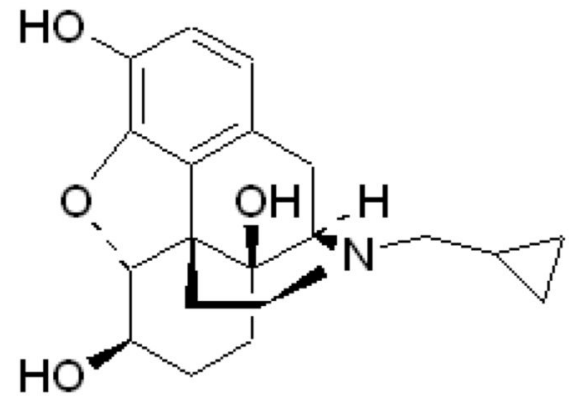

c. Naltrex ol free base, $\mathrm{pH} 8.5$ MW 343.4

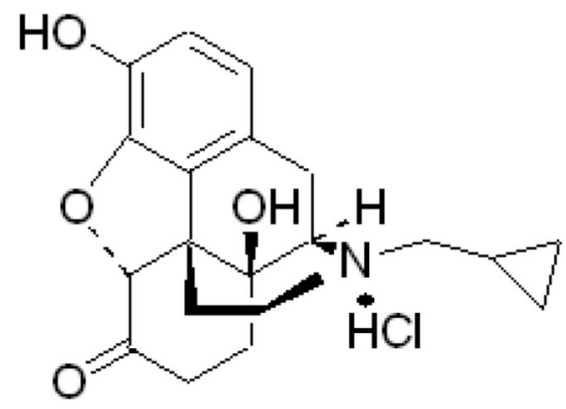

b. Naltr exone $\bullet \mathrm{HCl}$

MW 377.9

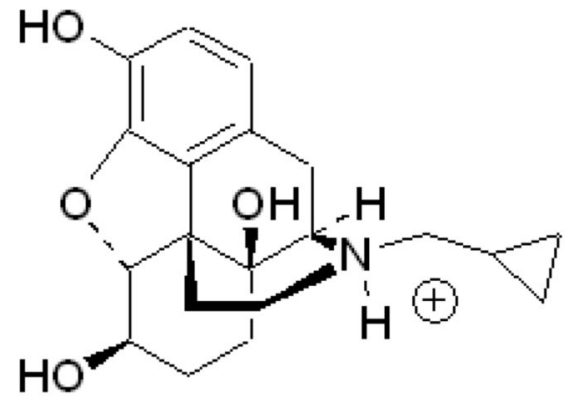

d. Naltrexol, $\mathrm{pH} 4.5$ MW 344.4

Fig. 1.

Structures of (a) naltrexone free base, (b) naltrexone $\mathrm{HCl}$, and 6- $\beta$-naltrexol at $\mathrm{pH}$ (c) 8.5 and (d) 4.5. 


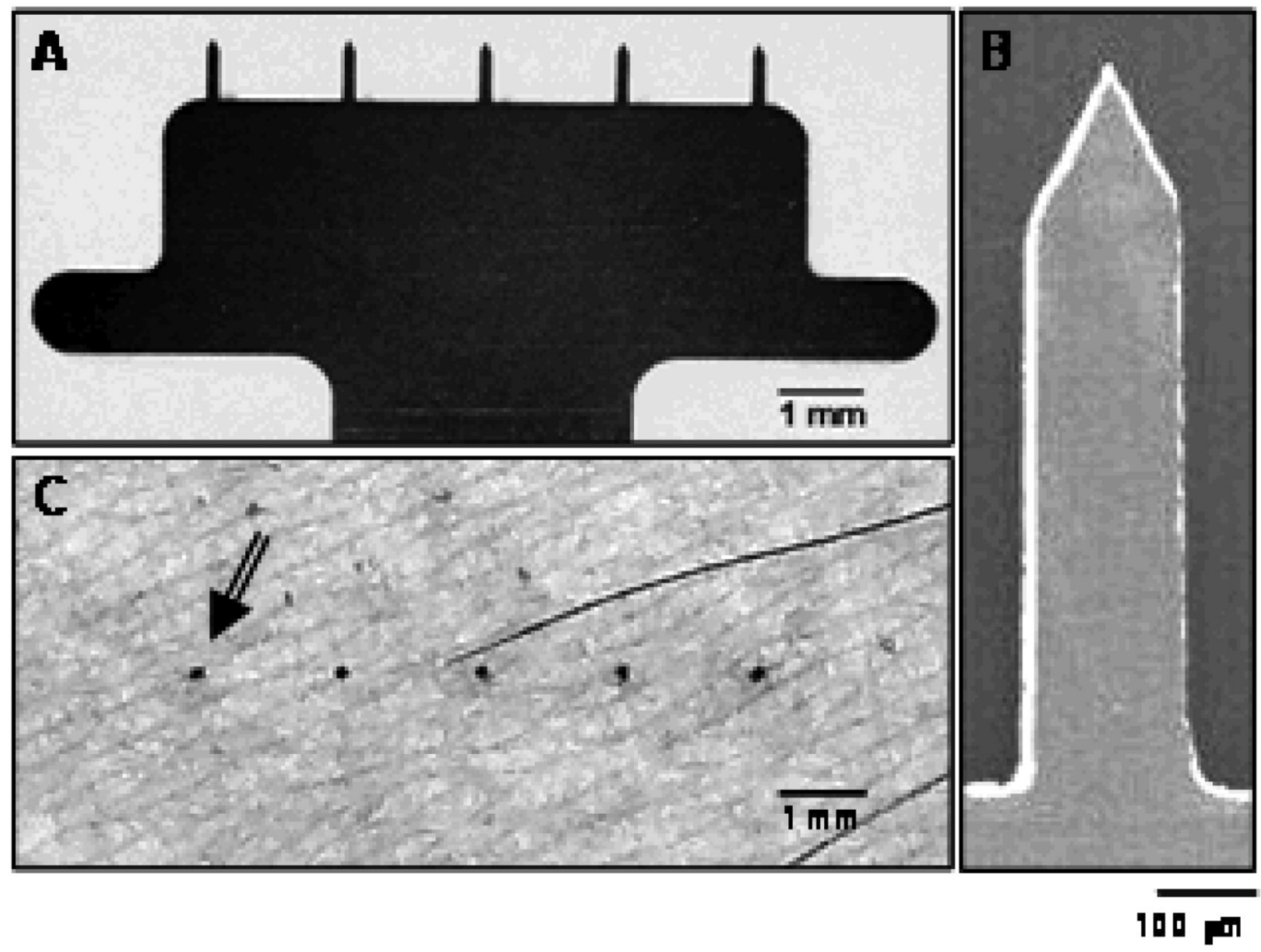

Fig. 2.

In-plane microneedles utilized in the in vitro skin permeation studies. (A) Brightfield micrograph of a row of five microneedles, (B) a scanning electron micrograph of a single microneedle of the row, and (C) brightfield micrograph of skin surface after piercing with a row of five microneedles and staining the skin with gentian violet. The arrow points to one of the five pores created by the microneedle row. 
(a)

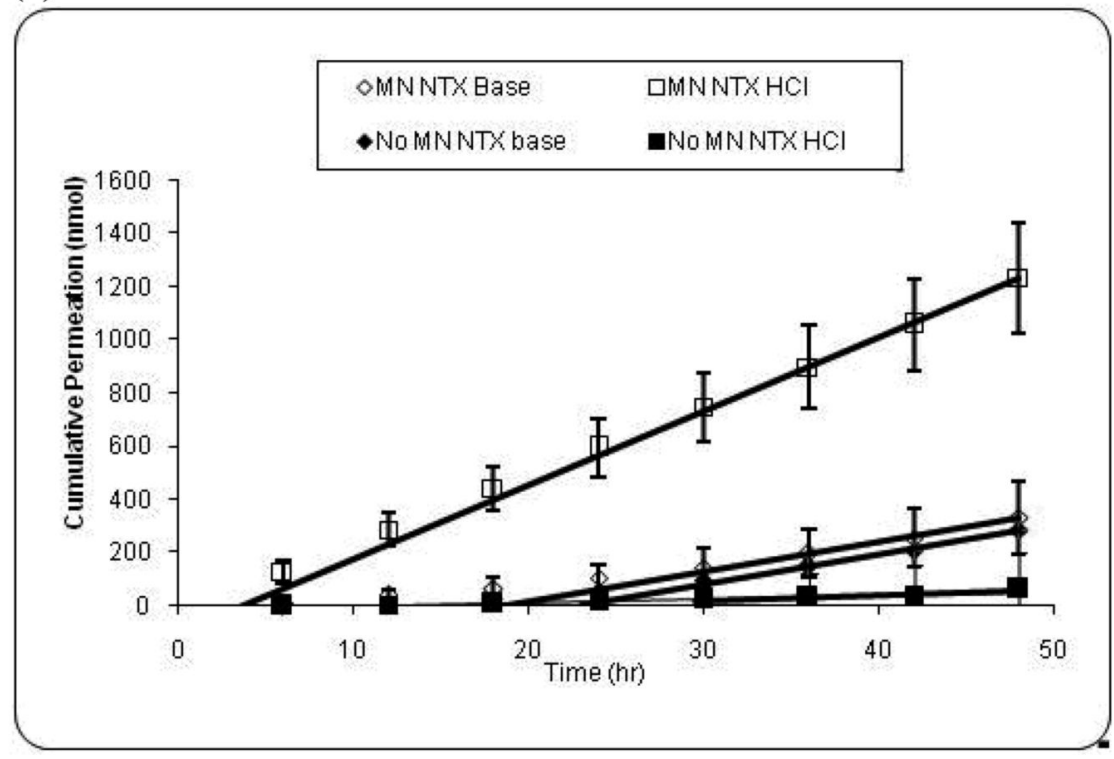

(b)

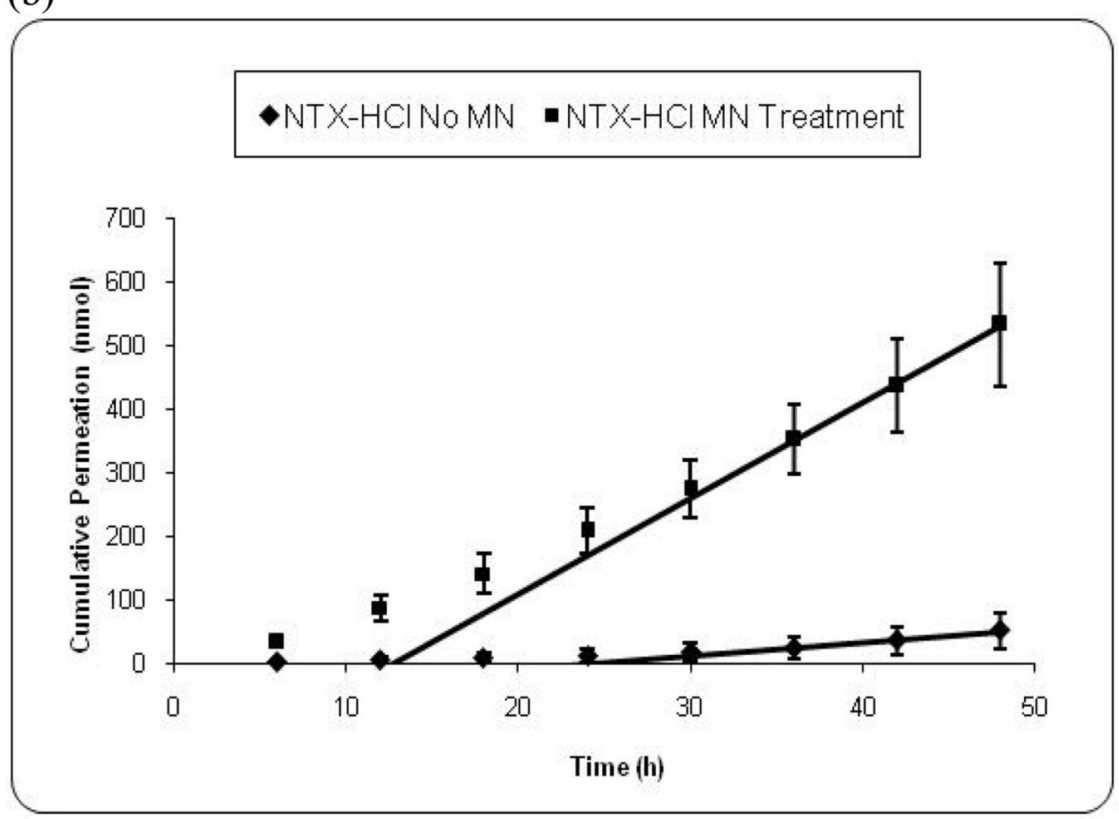

Fig. 3.

(a) Permeation and steady state flux profiles of microneedle-treated guinea pig skin with naltrexone free base $(n=10)(\diamond)$ and naltrexone hydrochloride salt $(n=12)(\square)$ and MNuntreated guinea pig skin with naltrexone free base $(\mathrm{n}=9)(\diamond)$ and naltrexone hydrochloride salt $(\mathrm{n}=10)(\mathbf{m})(\mathrm{p}<0.05)$. (b) Permeation and steady state flux profiles of human skin with naltrexone hydrochloride salt in presence $(n=4)(\boldsymbol{-})$ or absence $(n=3)(-)$ of MN's $(\mathrm{p}<0.05)$. 
(a)

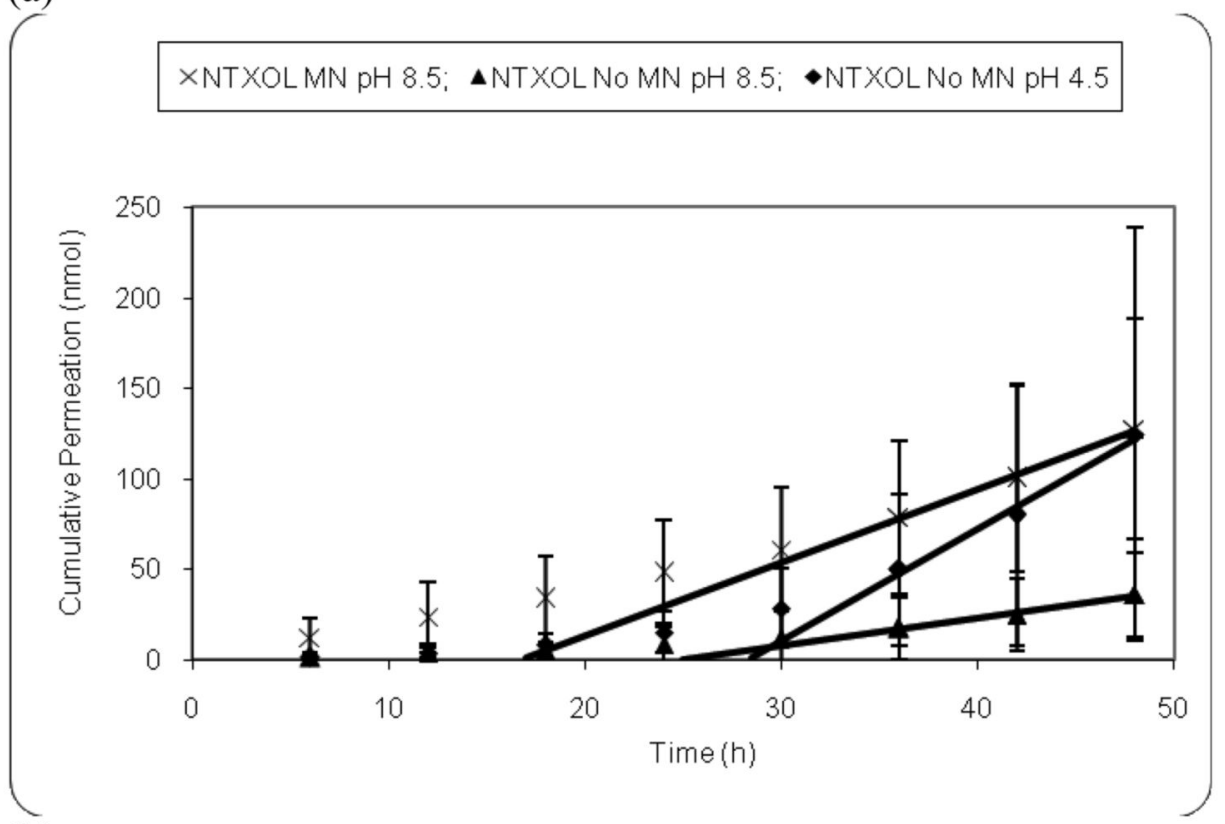

(b)

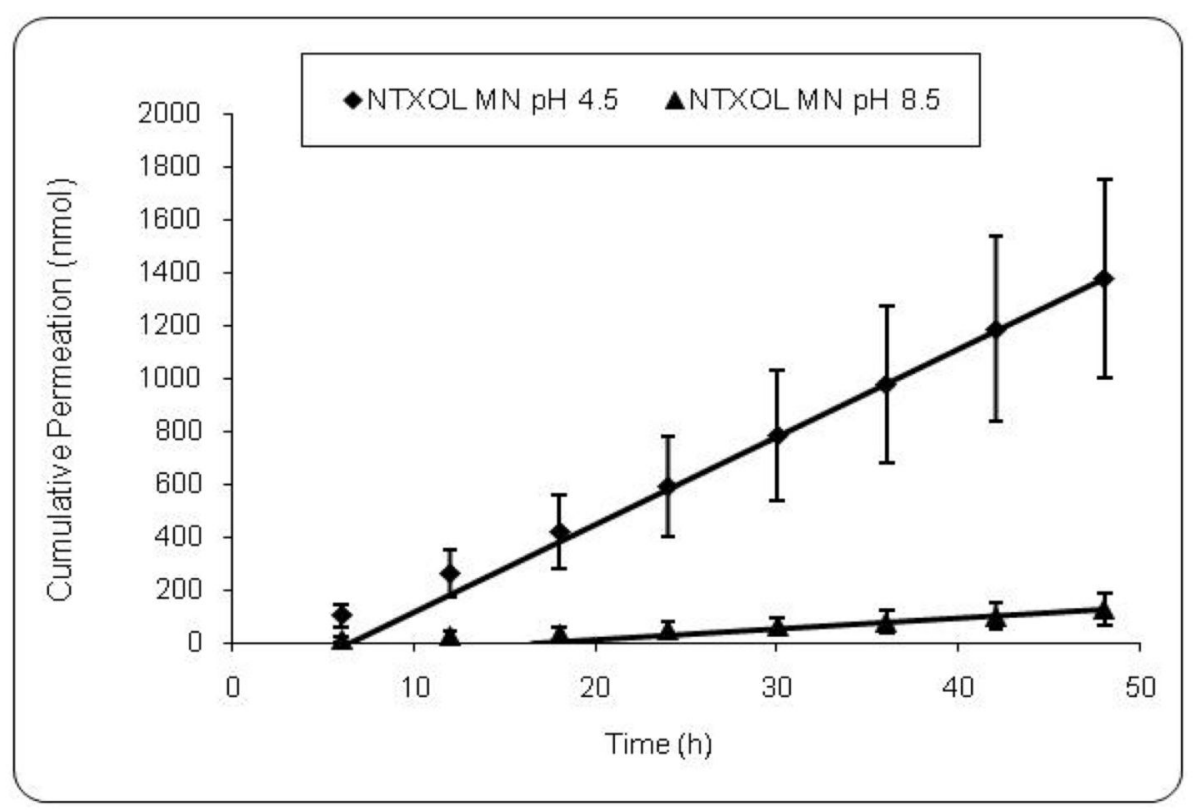

Fig. 4.

(a) Permeation and steady state flux profiles of MN-treated guinea pig skin with $(\times)(n=12)$ naltrexol free base, $\mathrm{pH} 8.5$ and untreated GP skin with naltrexol free base, $\mathrm{pH} 8.5(\mathrm{n}=9)(\boldsymbol{\Delta})$ and $\mathrm{pH} 4.5(\mathrm{n}=9)(\boldsymbol{)})$. (b) Permeation and steady state flux profiles of MN-treated guinea pig skin with naltrexol free base, $\mathrm{pH} 4.5(\mathrm{n}=12)(\boldsymbol{\square})$ and $\mathrm{pH} 8.5(\mathrm{n}=12)(\boldsymbol{\Delta})$. 
Banks et al.

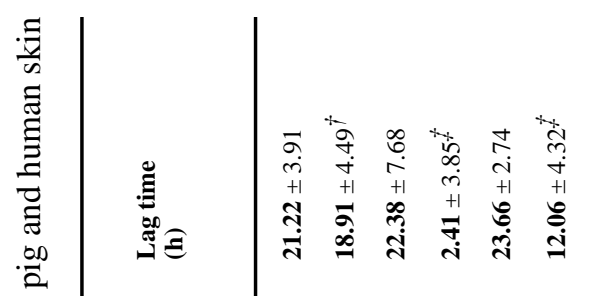

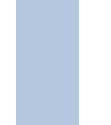

(1)

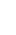

(1) 
Banks et al.

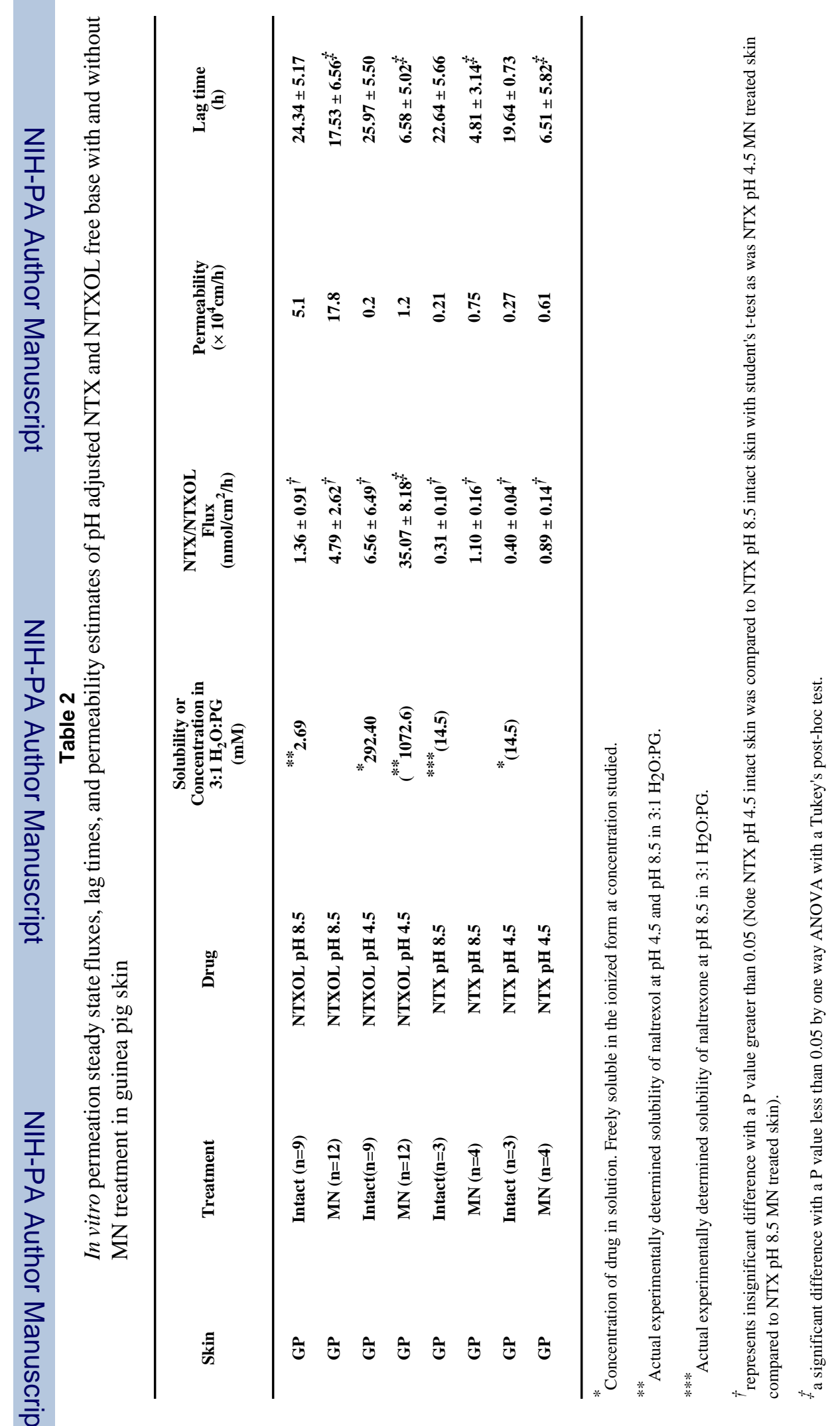

Pharm Res. Author manuscript; available in PMC 2009 October 5. 